\title{
Who cares about stem cells? We should!
}

Victor A. Ferraris, MD, PhD

See related article on pages 386-95.

Stem cells are truly remarkable. They have important characteristics, including self-renewal, in which they undergo unlimited asymmetric division. Somatic stem cells maintain and regenerate normal tissues by means of asymmetric and self-renewing divisions. Pluripotent stem cells proliferate seemingly indefinitely in culture, maintain a normal karyotype, and have the potential to generate any cell type in the body. They supply the cells that construct our adult bodies

From the Division of Cardiovascular and Thoracic Surgery, University of Kentucky, Lexington, Ky.

Disclosures: Author has nothing to disclose with regard to commercial support.

Received for publication May 10, 2015; accepted for publication May 12, 2015; available ahead of print June 6, 2015

Address for reprints: Victor A. Ferraris, MD, PhD, Division of Cardiovascular and Thoracic Surgery, A301, Kentucky Clinic, 740 South Limestone, Lexington, KY 40536-0284 (E-mail: Ferraris@earthlink.net).

J Thorac Cardiovasc Surg 2015;150:396-7

$0022-5223 / \$ 36.00$

Copyright (c) 2015 by The American Association for Thoracic Surgery

http://dx.doi.org/10.1016/j.jtcvs.2015.05.036 and replenish worn-out cells as we age. They renew themselves, resisting the powerful pull toward differentiation that overcomes most other cells in the body. Of note, defects in their proliferation capacity may result in cell aging or tumorigenesis. The observation that metastatic cancer cells exhibit experimental and clinical behaviors highly reminiscent of the classic properties of stem cells led researchers to search for and to characterize cancer stem cells (CSCs) believed to be implicated in tumorigenesis (http:// stemcells.nih.gov/info/Regenerative_Medicine/pages/2006 chapter9.aspx).

Self-renewal is arguably the most important and useful property of stem cells. Through this property, striking parallels can be found between stem cells and cancer cells. It is possible that tumors originate from the transformation of normal stem cells, similar signaling pathways may regulate self-renewal in stem cells and cancer cells, and collections of cancer cells may include CSCs-rare cells with indefinite potential for self-renewal that drive tumorigenesis. Evidence suggests that signaling pathways such as Shh, Notch, and Wnt, which regulate the self-renewal of stem cells, also play important roles in tumorigenesis.

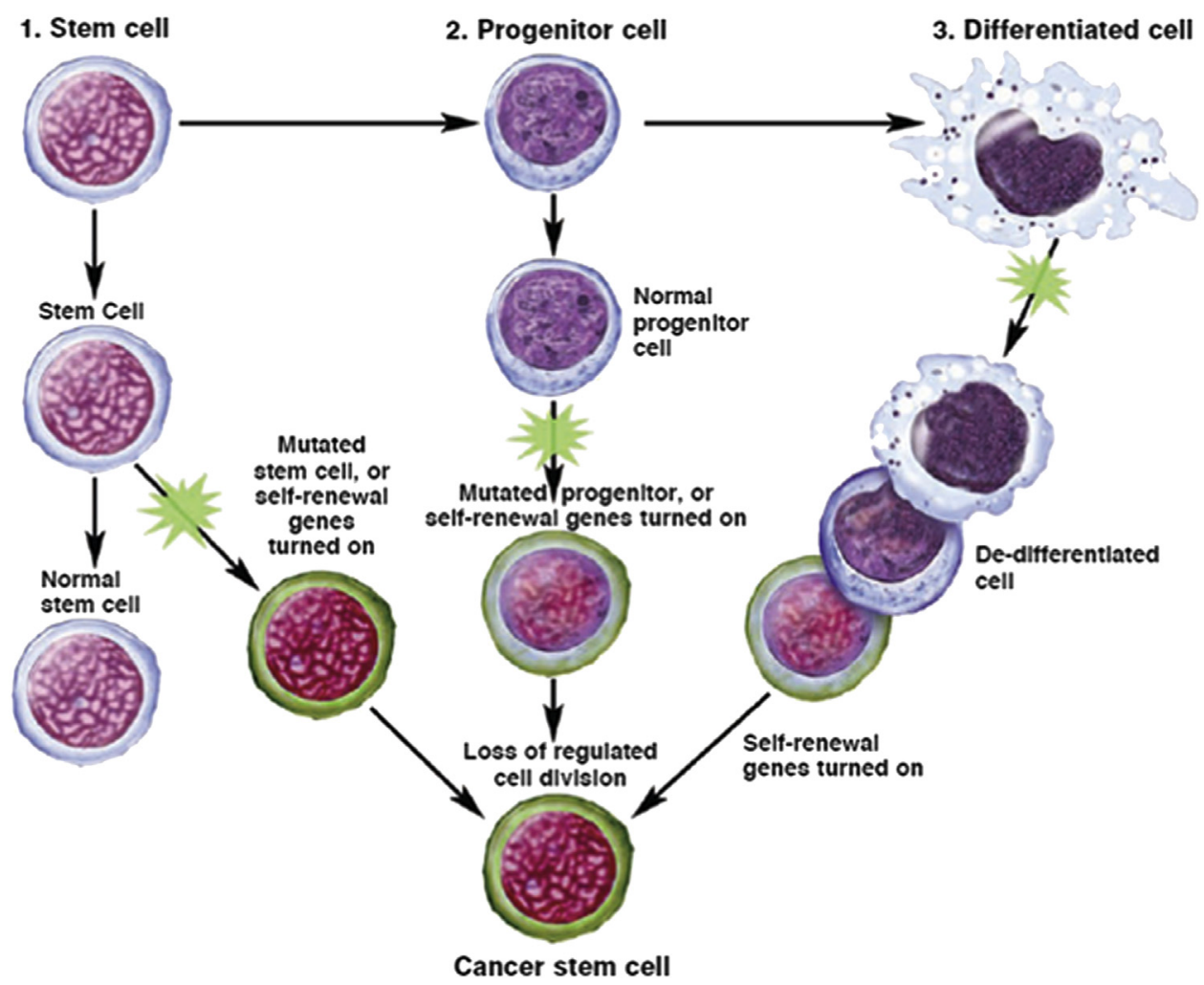

FIGURE 1. Three potential origins of cancer stem cells (http://stemcells.nih.gov/info/Regenerative_Medicine/pages/2006chapter9.aspx). 


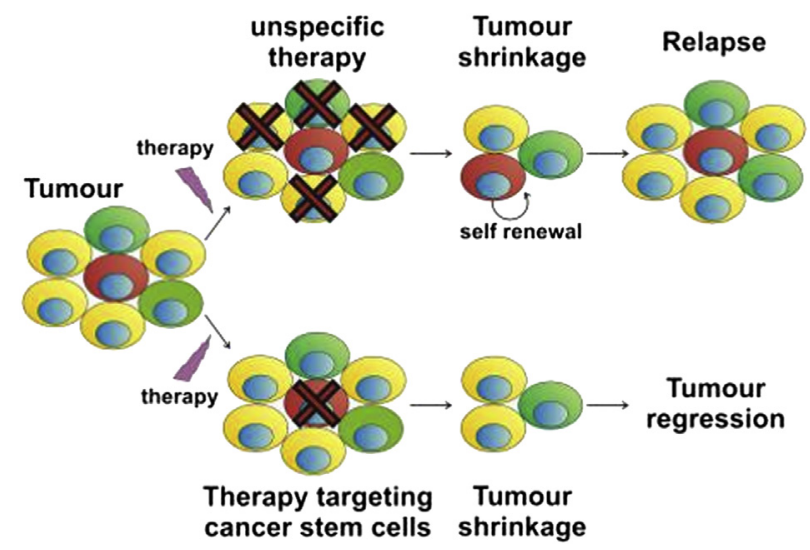

FIGURE 2. Cancer stem cell-specific therapy (http://www.eurostemcell. org/factsheet/cancer-disease-stem-cells).

Controversy exists regarding the origin of stem cells identified in tumors. In fact, unequivocal proof that pluripotent stem cells are the origin of CSCs is lacking. There are 3 potential origins of CSCs (Figure 1). Which of these origins is correct is the source of much debate. Characterization of stem cell-like populations uses cell-surface protein markers like CD133 and C-X-C chemokine receptor type 4 (CXCR4) to characterize putative CSCs in various tumors, including breast, colon, brain, pancreas, and prostate. These 2 markers are by no means the only cell surface markers of stem cells (http://en.wikipedia.org/wiki/Stem_cell_marker). In other cancers in which CSCs have yet to be identified, researchers are beginning to link established cell surface markers with stem cells. Lu and colleagues ${ }^{1}$ evaluate 2 membrane markers, CD133 and CXCR4, commonly expressed in hematopoietic and endothelial stem cells, to correlate CSCs with survival in an impressive number of patients with esophageal squamous cell cancer. As the authors point out, these markers have a putative role in tumor progression and metastasis in other nonesophageal cancers, but no previous studies have implicated these markers in esophageal cancer. The authors suggest that patients with positive CD133 and CXCR4 markers (ie, patients with evidence of increased CSCs activity) have worse survival. This intriguing result needs confirmation, but offers exciting possibilities, both for diagnosis and for selective treatment of esophageal cancer. Possibilities include targeting cell surface markers specific for CSCs with destructive antibodies and after success of this therapy with measurement of CD133 levels in the patient's bloodstream.

Most contemporary cancer treatments have limited selectivity - systemic therapies and surgeries remove or damage normal tissue in addition to tumor tissue. Therefore, these methods must be used judiciously to limit adverse effects. The really exciting component of the study of CSCs is the potential for selective diagnosis and treatment of tumors using therapy directed at stem cells as opposed to nonselective treatments with the potential for destructive non-cancerrelated injury (Figure 2).

One strength of the article by $\mathrm{Lu}$ and colleagues ${ }^{1}$ is the large volume of patients with esophageal squamous cell cancer and their impressive clinical staging and survival follow-up. The authors attempted to build on this clinical follow-up by assessing laboratory correlates of survival. Ultimately, if measurement of these 2 CSC-related proteins proves to have some clinically meaningful prognostic ability, then measurements would need to be done before institution of neoadjuvant therapy. These markers might ultimately give some indication of the success of neoadjuvant therapy in tumor regression. It is important to understand how measurements of CSCs might fit into the clinical prognostic and diagnostic picture, but the future for indicators of CSCs is exciting.

So why should we care about stem cells? CSCs may hold the key to diagnosis and treatment of one of the most dismal diseases that thoracic surgeons treat. Surgeons should jump on the stem cell bandwagon and follow the lead of the authors. An honest assessment of our treatment of esophageal squamous cell cancer has to conclude that we have failed. The 5-year survival is still less than $15 \%$ and has not changed much in the last 50 years. Do stem cells hold the key to change this prognosis? I hope so.

\section{Reference}

1. Lu C, Xu F, Gu J, Yuan Y, Zhao G, Yu X, et al. Clinical and biological significance of stem-like CD133+CXCR4+ cells in esophageal squamous cell carcinoma. $J$ Thorac Cardiovasc Surg. 2015;150:386-95. 